\title{
Loss of CD73-mediated extracellular adenosine production exacerbates inflammation and abnormal alveolar development in newborn mice exposed to prolonged hyperoxia
}

\author{
Huiling $\mathrm{Li}^{1}$, Harry Karmouty-Quintana ${ }^{2}$, Ning-Yuan $\mathrm{Chen}^{2}$, Tingting Mills ${ }^{2}$, Jose Molina ${ }^{2}$, Michael R Blackburn ${ }^{2}$ and \\ Jonathan Davies ${ }^{1}$
}

\begin{abstract}
BACKGROUND: Hyperoxic lung injury is characterized by cellular damage from high oxygen concentrations that lead to an inflammatory response and it disrupts normal alveolarization in the developing newborn lung. Adenosine is a signaling molecule that is generated extracellularly by ecto-5'-nucleotidase (CD73) in response to injury. Extracellular adenosine signals through cell surface receptors and has been found to have a protective role in acute injury situations; however, chronic elevations have been associated with detrimental changes in chronic lung diseases. We hypothesized that hyperoxia-induced lung injury leads to CD73-mediated increases in extracellular adenosine, which are detrimental to the newborn lung.
\end{abstract}

METHODS: $\mathrm{C} 57 \mathrm{BI} / 6$ and $\mathrm{CD}^{-1-}$ mice were exposed to 95\% oxygen, $70 \%$ oxygen, or room air. Adenosine concentration and markers of pulmonary inflammation and lung development were measured.

RESULTS: Exposure to hyperoxia caused pulmonary inflammation and disrupted normal alveolar development in association with increased pulmonary adenosine levels. Loss of CD73-mediated extracellular adenosine production led to decreased survival with exposure to 95\% oxygen, and exacerbated pulmonary inflammation and worsened lung development with 70\% oxygen exposure.

CONCLUSION: Exposure to hyperoxia causes lung injury associated with an increase in adenosine concentration, and loss of CD73-mediated adenosine production leads to worsening of hyperoxic lung injury.

B ronchopulmonary dysplasia (BPD) remains a significant cause for morbidity and mortality in premature neonates (1). BPD occurs in $\sim 1 / 3$ of infants less than $1,000 \mathrm{~g}$ and is associated with worse developmental outcomes, increased re-hospitalization, and significant costs (2).

Oxygen supplementation is one of the primary respiratory supports for premature neonates. However, high levels of oxygen exposure cause pulmonary inflammation and injury and contribute to the development of BPD (3). Along with inflammatory changes, there is widespread disruption of pulmonary development resulting in simplified and enlarged alveoli-the hallmark features of BPD histopathology (1). The altered developmental signaling observed in BPD includes changes in growth factors such as insulin-like growth factor 1 (IGF-1) and keratinocyte growth factor (KGF) that disrupt normal alveolarization (4).

There is growing evidence that adenosine signaling is a key regulatory pathway contributing to the development of BPD. Adenosine is recognized as one of the important extracellular signaling molecules that regulates alveolar development as well as pulmonary inflammation and repair processes following injury (5-8). Adenosine is a nucleoside that is generated extracellularly in response to cellular injury via the dephosphorylation of extracellular adenosine triphosphate (ATP) by the enzymes ectonucleoside triphosphate diphosphohydrolase-1 (CD39) and ecto-5'-nucleotidase (CD73). CD73 dephosphorylates AMP to adenosine and is the final step in the production of extracellular adenosine. Extracellular adenosine binds to a family of G-proteincoupled receptors (Adora1, Adora2A, Adora2B, and Adora3) on the cell surface to activate a cascade of intracellular cellular-response pathways, primarily through the activation or inhibition of adenylate cyclase and the subsequent alteration of intracellular cyclic adenosine monophosphate levels $(5,8)$.

During acute lung injury, elevated adenosine levels activate anti-inflammatory and tissue-protective mechanisms that protect the lung $(5,9)$. Acute adenosine signaling blunts the production of multiple cytokines, decreases inflammatory cell infiltration, and preserves pulmonary vascular barrier function (10). However, sustained adenosine signaling activate pro-inflammatory pathways with increased cytokine production and inflammatory cell infiltration $(8,11)$. Finally, elevated adenosine levels in the developing lungs of adenosine deaminase (ADA) knock out newborn mice lead to disruption of normal alveologenesis (6). Administration of exogenous

'Division of Neonatal-Perinatal Medicine, Department of Pediatrics, Baylor College of Medicine, Houston, Texas; ${ }^{2}$ Department of Biochemistry and Molecular Biology, The University of Texas-Houston Medical School, Houston, Texas. Correspondence: Jonathan Davies (Jd4@bcm.edu)

Received 14 March 2017; accepted 20 June 2017; advance online publication 23 August 2017. doi:10.1038/pr.2017.176 
ADA metabolizes extracellular adenosine, decreases adenosine signaling, and rescues the normal phenotype, suggesting that elevations in adenosine are causative in disrupting alveologenesis (6).

Studies to investigate the potential role of adenosine in BPD are limited. Adenosine receptor polymorphisms in neonates correlate with an increased susceptibility to BPD (12). In addition, serum ADA levels were elevated in premature infants who develop BPD with a positive relationship between levels of ADA and BPD severity (13). Finally, caffeine, an adenosine receptor antagonist used to treat apnea of prematurity, decreases the risk of BPD in very low birthweight infants (14). Recent studies suggest that caffeine may be working directly on the lung to decrease adenosine signaling and alter pathways that lead to BPD (15-18).

We hypothesized that exposure to high levels of oxygen to induce pulmonary injury will cause an increase in pulmonary extracellular adenosine, and genetic deletion of CD73 to decrease extracellular adenosine production will improve inflammation and alveolar development in a hyperoxic murine model of BPD.

\section{METHODS}

\section{Animal Model in Hyperoxia}

Newborn C57BL/6 mice were used as wild-type controls. CD73 ${ }^{-/-}$ mice were generated on a $\mathrm{C} 57 \mathrm{Bl} / 6$ background and genotyped as previously reported (19). Newborn pups from multiple litters were pooled and then randomly distributed to exposure to room air $(21 \%$ $\mathrm{O}_{2}$ ), $95 \%$ oxygen, or $70 \%$ oxygen for 14 days starting between 12 and $24 \mathrm{~h}$ after birth. The litter size was limited to six to eight pups per litter. Nursing dams were rotated between room air and hyperoxia every $24 \mathrm{~h}$. Oxygen exposure occurred in an A-Chamber (Biospherix, Lacona, NY) equipped with ProOx 110 controller (Biospherix) in the same room as room air control animals. Oxygen concentrations were monitored continuously and maintained at 70 or $95 \pm 2 \%$ in the chamber at all times. The room was regulated with a 12-h light-dark cycle. Food and water were supplied ad libitum. Maintenance and care of animals were in accordance with the guidelines set by the Animal Welfare Committee (AWC) at the University of Texas Health Science Center (UTHSC) at Houston, and all experiments were approved by the AWC at the UTHSC at Houston.

\section{Bronchoalveolar Lavage Fluid Collection and Lung Harvest}

Bronchoalveolar lavage fluid (BALF) was obtained during terminal procedure as previously described $(7,9)$. Briefly, at the time of sample collection, mice were anesthetized with avertin, and their lungs were lavaged four times with $0.1 \mathrm{ml}$ phosphate-buffered saline containing $10 \mu \mathrm{m}$ dipyridamole (Sigma-Aldrich, St. Louis, MO), $10 \mu \mathrm{m}$ ADAinhibitor deoxycoformycin (dCF, R\&D Systems Inc., Minneapolis, $\mathrm{MN}$ ), and $10 \mu \mathrm{M} \alpha \beta$-methylene $\mathrm{ADP}$, which pooled $\sim 0.3 \mathrm{ml}$ fluid. A sample was separated for cell count determination using a hemocytometer. The remaining BALF was centrifuged and the supernatant stored for further analyses. Cell pellets were resuspended and aliquots were cytospun onto slides and stained with Diff-Quick (Dade Behring, Deerfield, IL) for cellular differential determination. After lavage, the pulmonary vasculature was perfused with phosphate-buffered saline by direct injection into the right ventricle. Lungs were then collected for either RNA and protein or for histology. Lungs taken for RNA and protein analysis were removed and frozen in liquid nitrogen. Whole lungs were pulverized using a BioPulverizer (Biospec, Bartlesville, $\mathrm{OK}$ ) and separated into aliquots for RNA and protein extraction. Those lungs for histology were inflated with constant pressure of $20 \mathrm{cmH}_{2} \mathrm{O}$ and fixed in formalin for $24 \mathrm{~h}$. The lungs were processed and paraffin-embedded.
Five-micron sections were cut and used for histology. All lungs were processed in the same manner according to protocol to assure quality and prevent bias.

\section{Adenosine Measurement}

Adenosine concentrations in BALF supernatant were measured using high-performance liquid chromatography as previously described (9). One hundred microliters of BALF supernatant was loaded in the high-performance liquid chromatography meter per reading and the flow rate was set at $1.5 \mathrm{ml} / \mathrm{min}$. The representative peaks were identified and quantitated by running known external standard curves.

\section{Lung Histology and Morphometry}

Mouse lungs were collected and fixed in $10 \%$ formaldehyde for at least $24 \mathrm{~h}$. The lungs were then dehydrated, paraffin-embedded, and sectioned $(5 \mu \mathrm{m})$. The sections were rehydrated and stained with hematoxylin and eosin (Sigma-Aldrich) according to the manufacturer's instructions. Alveolar development was evaluated using the mean linear intercept (MLI) and radial alveolar counts (RAC) as previously described $(20,21)$. Areas were randomly chosen and photographed at $\times 20$ magnification for MLI and $\times 10$ magnification for RAC. For MLI, areas with large airways and blood vessels were not included. For each morphometric analysis, 10-15 areas per slide were quantitated and averaged per slide. Image acquisition and analysis were carried out in a blinded manner to prevent bias.

\section{Cell Culture}

Mouse MLE12 (transformed mouse alveolar epithelial type II cell line) cells (ATCC, Manassas, VA) were cultured in RPMI Media 1640 (Sigma) containing $10 \%$ fetal bovine serum and antibiotics. For hyperoxia exposure, the cells were exposed to 35,55 , or $75 \%$ oxygen and $5 \% \mathrm{CO}_{2}$ for 24,48 , or $72 \mathrm{~h}$.

\section{Western Blotting and Enzyme-Linked Immunosorbent Assay Analysis of interleukin-6}

Protein was extracted from pulverized lungs or cells using RIPA lysis buffer (50 mm Tris- $\mathrm{HCl} \mathrm{pH} 7.4,150 \mathrm{~mm} \mathrm{NaCl}, 1 \% \mathrm{NP}-40$ ) containing a protease inhibitor cocktail (Thermo Fisher Scientific, Fair Lawn, NJ). Equal amounts of protein were separated on sodium dodecyl sulfate-polyacrylamide gel electrophoresis and transferred to nitrocellulose membranes. The membranes were blocked with 5\% (w/v) nonfat milk, washed with Tris-buffered saline-Tween-20, and incubated with primary anti-CD73 antibodies (1:1,000, goat polyclonal, Santa Cruz), anti-IGF-1 antibodies (1:500, goat polyclonal, Santa Cruz), anti-KGF antibodies (1:1,000, rabbit polyclonal, Abcam), anti-actin antibodies (1:10,000, mouse monoclonal, Sigma), and anti-GAPDH antibodies (1:20,000 mouse monoclonal, Thermo Fisher Scientific) overnight at $4^{\circ} \mathrm{C}$. Membranes were then rinsed, incubated with corresponding secondary antibodies conjugated to horseradish peroxidase (Jackson ImmunoResearch, West Grove, PA) for $1 \mathrm{~h}$ at room temperature, and developed with HyGLO Chemiluminescent horseradish peroxidase Antibody Detection Reagent (Denville Scientific Inc., Holliston, MA). The Protein isolated from lung lysate was also processed using a mouse interleukin (IL)-6 enzyme-linked immunosorbent assay kit (BD Biosciences, San Jose, CA).

\section{Real-Time PCR}

Total RNA was isolated from frozen lung tissue using Trizol reagent (Life Technologies). RNA samples were then DNAse-treated (ArticZymes, Tromso, Norway) and subjected to quantitative realtime RT-PCR. Transcript levels for mouse CD73 were determined by normalization to $\beta$-actin and are presented as the mean normalized transcript levels using the comparative Ct method $(2 \Delta \Delta \mathrm{Ct})$. 


\section{CD73 in newborn hyperoxic lung injury $\quad$ Articles}

Statistical Analysis

Results are reported as means \pm SEM. Comparison between groups used two-tailed Student's $t$-test. Comparison among groups used two-way ANOVA followed by Tukey post tests for comparisons against control conditions using GraphPad version 5. Significance was assigned for $P<0.05$.

\section{RESULTS}

\section{Adenosine Levels are Elevated in Newborn Mice Exposed to} 95\% Oxygen and Loss of CD73 Decreases Survival

We hypothesized that cellular injury caused by exposing newborn mouse pups to a high oxygen concentration would produce elevations of extracellular adenosine levels. To test our hypothesis, we used a severe hyperoxia model of BPD, which has been used to interrogate regulatory pathways important in the pathophysiology of BPD (22). In this model, we placed newborn wild-type $\mathrm{C} 57 \mathrm{Bl} / 6$ pups in room air or 95\% oxygen for 14 days. At the end of exposure, the mice were killed for evaluation of pulmonary inflammation, lung development, and adenosine concentration. BALF from $95 \%$ oxygen-exposed mice had increased cell counts when compared with their room air-exposed controls (Figure 1b), and lung histology demonstrated disruption of normal alveolar development quantitated by increased MLI and decreased RAC in 95\% oxygen-exposed mice when compared with that in the room air-exposed controls (Figure 1a). These inflammatory changes and disruption of alveolar development were associated with a fourfold increase in adenosine concentration in hyperoxic-exposed mice when compared with that in the room air controls (Figure 1c).
To evaluate the role of elevated adenosine levels in this severe hyperoxia model, we exposed $\mathrm{CD}_{73}^{-/}$mice to room air or $95 \%$ oxygen. CD73 is the membrane-bound enzyme that performs the last step of dephosphorylation of AMP in generation of extracellular adenosine. CD73 mice are consequently unable to generate extracellular adenosine from ATP that is released during cellular injury. Survival of $\mathrm{CD} 73^{-/-}$ mice was significantly decreased in 95\% oxygen when compared with the wild-type controls, leading to a median survival of only 6 days and death of all animals by day 11 of exposure (Figure 1d). This finding indicates that CD73 generation of adenosine is protective in hyperoxic lung injury in newborn mouse pups.

\section{Seventy percent Oxygen Exposure Induced Inflammation, Disrupted Alveolar Development, and Increased Adenosine Levels}

The increased mortality in $\mathrm{CD}^{-/-}$mice in $95 \%$ oxygen precluded our ability to interrogate the mechanistic role of CD73 in hyperoxic lung injury. Therefore, we utilized a less severe mouse model of BPD (23) using exposure to a lower oxygen concentration (70\%) for 14 days to evaluate the role of CD73-mediated extracellular adenosine in hyperoxic lung injury in newborn mice. At the conclusion of exposure, the mice were killed for evaluation of pulmonary inflammation, lung development, and adenosine concentration. Survival for both wild-type and $\mathrm{CD}^{-/-}$mice in this model was $>93 \%$ for both room air and 70\% oxygen exposure over 14 days, with no difference between groups. BALF from $70 \%$ oxygen- a
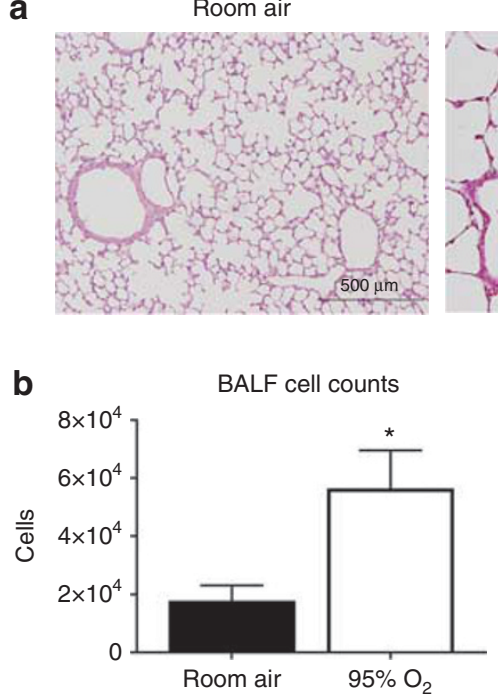

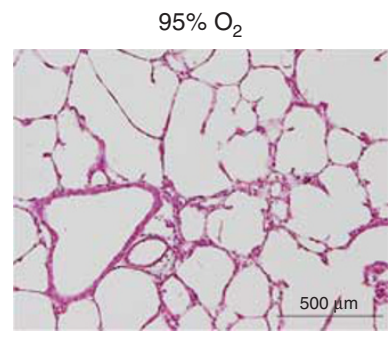

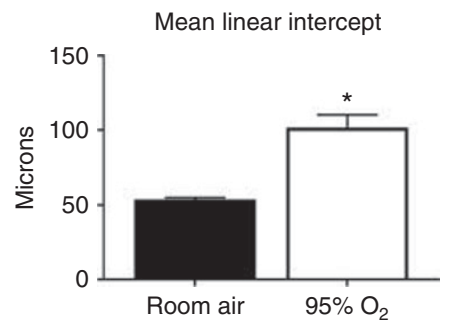

Radial alveolar count

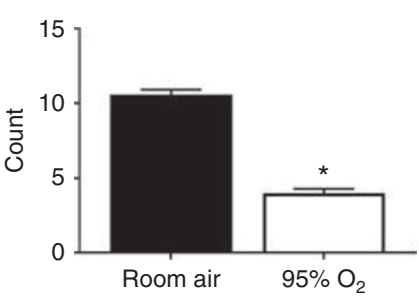

C

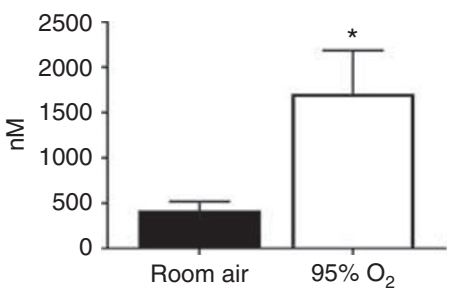

d

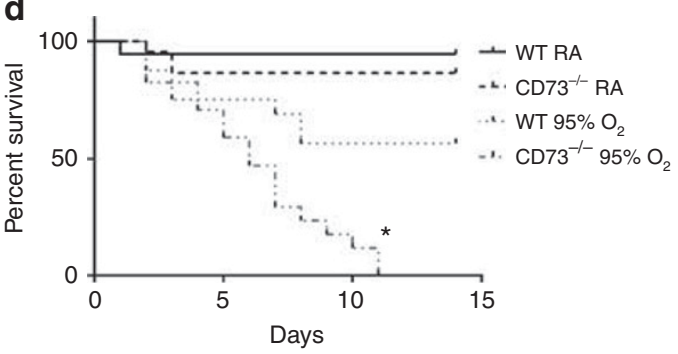

Figure 1. Adenosine levels are elevated in $95 \%$ hyperoxia and loss of CD73 worsens survival in hyperoxia. Wild-type mice were exposed starting $12-24 \mathrm{~h}$ after birth to either room air or $95 \%$ hyperoxia $\left(95 \% \mathrm{O}_{2}\right)$ for 14 days. BALF analysis showed increase in cell count (b), indicating an increased inflammatory cell infiltration. (a) Representative histology (original magnification $\times 10$ ) demonstrates marked abnormalities in alveolar development with decreased alveolar number, larger alveolar spaces, and increased cellularity. Analysis demonstrated enlarged alveolar spaces with a significant increase in the mean linear intercept and decreased alveoli number with a significant decrease in radial alveolar count in $95 \%$ oxygen-exposed animals ( $n=4$ per group). (c) BALF adenosine concentration was found to be elevated approximately fourfold in mice exposed to $95 \%$ oxygen ( $n=6$ for room air, 8 for hyperoxia). (d) Survival curve demonstrates $56 \%$ survival of wild-type mice in $95 \%$ oxygen (WT $95 \% \mathrm{O}_{2}$ ), but $0 \%$ survival in $\mathrm{CD}^{-/-}$mice in $95 \%$ oxygen $\left(\mathrm{CD}^{-1 /} 95 \% \mathrm{O}_{2}\right)$ with the median survival of 6 days. ${ }^{*} P \leq 0.05$. BALF, bronchoalveolar lavage fluid; WT, wild type. 


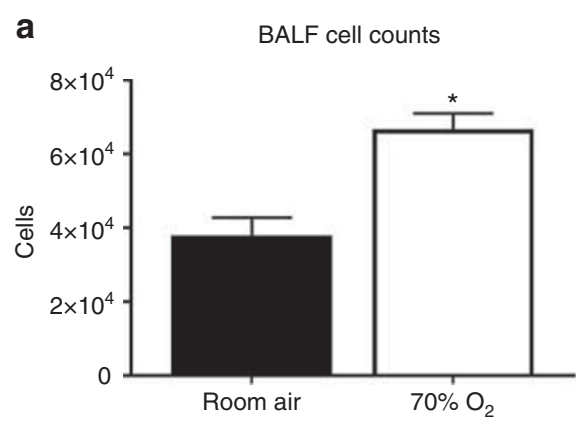

b BALF adenosine levels
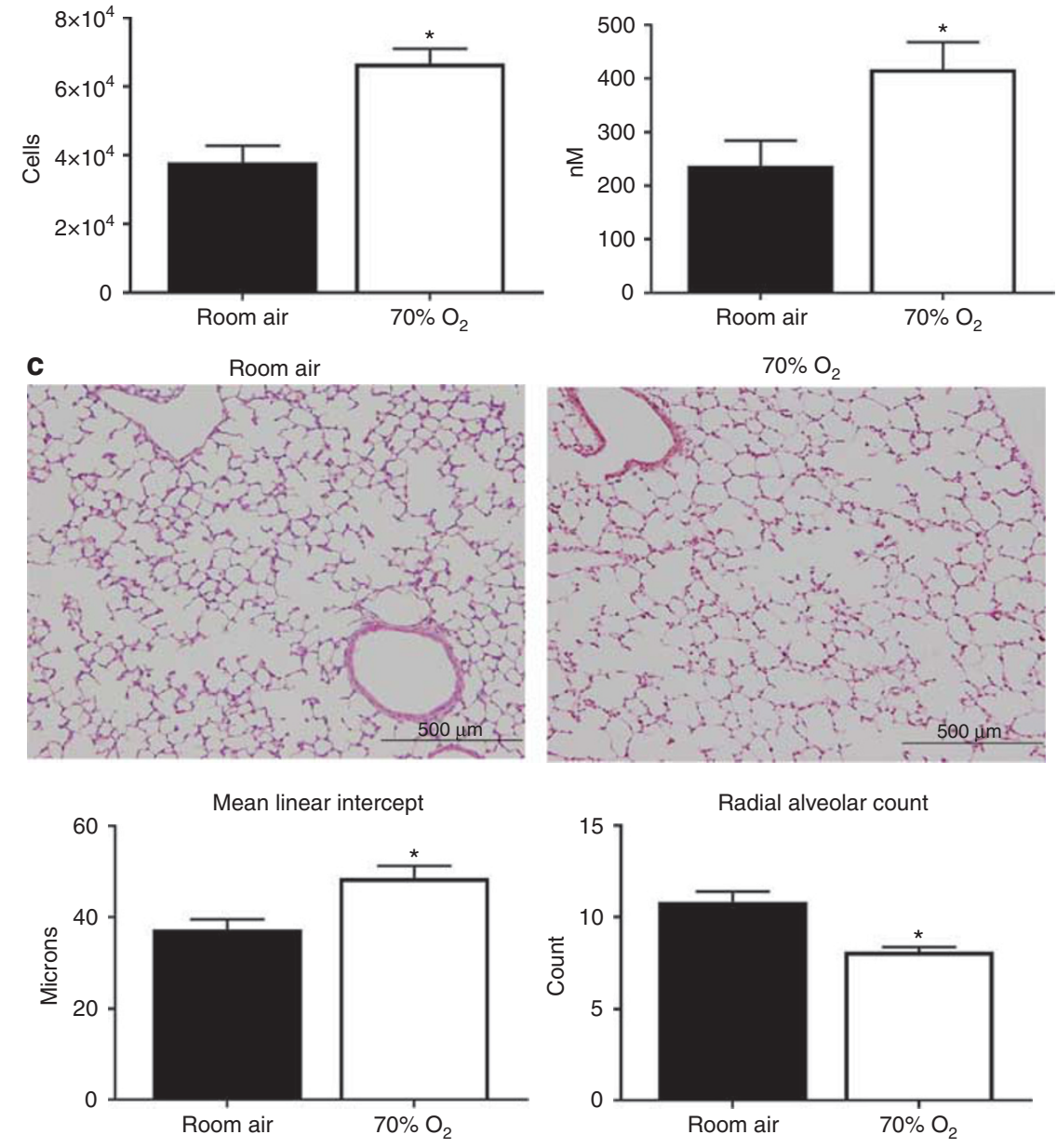

Figure 2. Adenosine levels are elevated in 70\% hyperoxia. Wild-type mice were exposed starting $12-24 \mathrm{~h}$ after birth to either room air or $70 \%$ hyperoxia $\left(70 \% \mathrm{O}_{2}\right)$ for 14 days. BALF analysis showed an increase in cell count (a), indicating increased inflammatory cell infiltration. (c) Representative H\&E-stained histology (original magnification $\times 10$ magnification) demonstrates alveolar simplification with enlarged alveolar spaces with a significant increase in the mean linear intercept and decreased alveoli number with a significant decrease in radial alveolar count in $70 \%$ oxygen-exposed animals when compared with room-air-exposed animals. (b) BALF adenosine concentration was found to be elevated 1.8-fold in mice exposed to $70 \%$ oxygen. ${ }^{*} P \leq 0.05 . N=8$ for room air and 28 for hyperoxia. BALF, bronchoalveolar lavage fluid; H\&E, hematoxylin and eosin.

exposed wild-type C57Bl/6 mice again had increased cell counts when compared with that from room air-exposed controls (Figure 2a). Lung histology revealed alveolar simplification, with enlarged alveoli demonstrated by an increase in MLI and a decrease in RAC (Figure 2c). These changes were associated with a 1.8 -fold increase in BALF adenosine concentration (Figure 2b).

\section{Hyperoxia Leads to a Decrease in CD73 Transcript and Protein Levels}

CD73 is tightly regulated in response to lung injury in a variety of lung diseases, and measurements of expression have proven to be a useful approach in understanding the regulation of adenosine synthesis and signaling during disease processes $(9,24)$. The changes in CD73 are largely driven by hypoxia and transcriptional regulation by hypoxia-inducible factors (HIF) (25). Hyperoxia, on the other hand, has been shown to blunt the protective actions of HIF in the lung (24), but still can upregulate CD73 and Adora2b in adult mouse hyperoxic lung injury (9). We hypothesized that we would see a similar increase in CD73 in newborn mice exposed to hyperoxia. Whole-lung lysates from wild-type mice exposed to room air or oxygen were obtained before exposure to oxygen (P1) and during room air or $70 \%$ oxygen exposure (P3, P8, and P15). Expression of CD73 in wild-type mice in room air showed a progressive increase in mRNA levels from P1 to P8, but then a decrease by P15 (Figure 3a). Hyperoxiaexposed mice also had increasing CD73 transcript levels to P8, but at P8 the level of CD73 mRNA was significantly less than room air controls. At P15, the transcript levels decreased as comparable to the levels in room air controls. Western blot analysis demonstrates a similar trend with an increase in CD73 protein in wild-type animals in room air from P8 to P15 (Figure 3b). However, hyperoxia-exposed mice had 


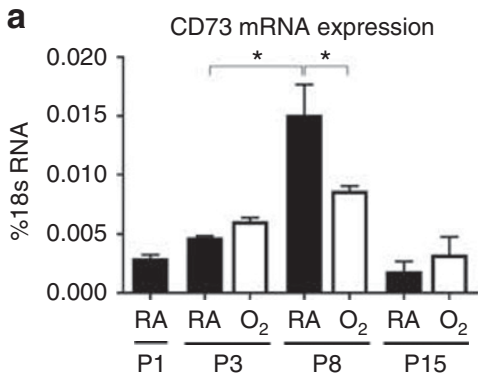

C

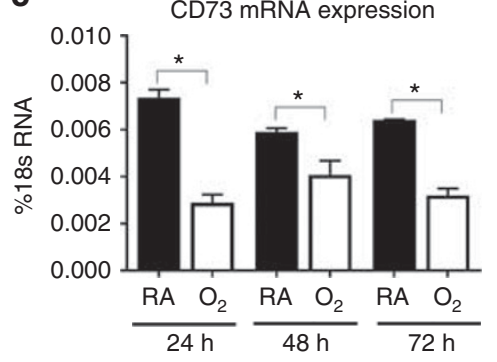

b
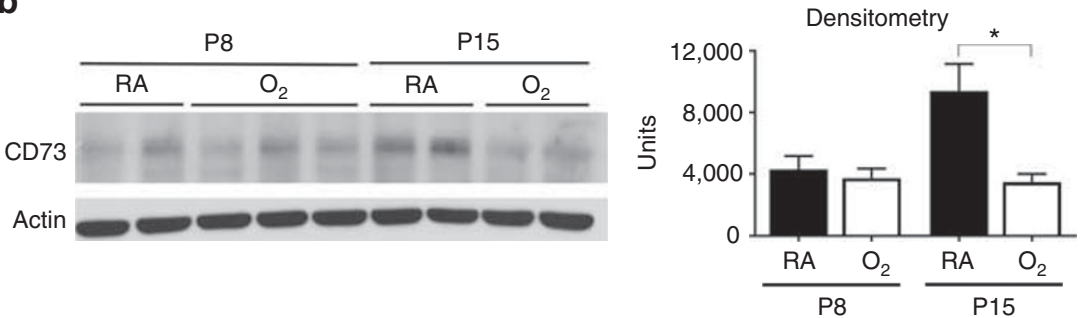

d

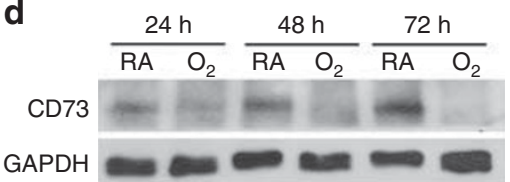

e

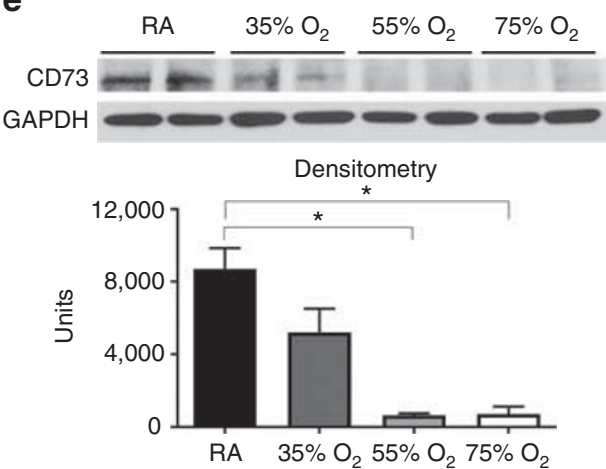

Figure 3. Oxygen exposure decreases CD73 transcript and protein levels in mice and MLE-12 cells. (a) Quantitative PCR analysis of whole-lung lysates from wild-type mice showed increasing transcript levels in room air from P3 to P8, but then a decrease at P15. Wild-type mice exposed to $70 \%$ oxygen $\left(\mathrm{O}_{2}\right)$ showed decreased $\mathrm{CD} 73$ transcript levels at P8 when compared with those exposed to room air. $N=3-8$ mice per time point per oxygen exposure. (b) Western analysis showed comparable levels of CD73 protein at P8, but decreased levels at P15 in mice exposed to $70 \%$ oxygen. (c) Quantitative PCR analysis of MLE- 12 cells exposed to room air or $75 \%$ oxygen $\left(\mathrm{O}_{2}\right)$ for 24,48 , and 72 h showed decreased CD73 transcript levels at all time points in oxygen-exposed cells. (d) Western blot analysis of CD73 in oxygen-exposed MLE-12 cells shows decreased protein at 24,48 , and $72 \mathrm{~h}$ of exposure to $75 \%$ oxygen. (e) Western blot analysis of CD73 protein in room air, $35 \%$ oxygen, $55 \%$ oxygen, and $75 \%$ oxygen for $48 \mathrm{~h}$ demonstrates decreased protein with increasing oxygen concentration. ${ }^{*} P \leq 0.05$.

significantly less CD73 when compared with room air controls. These findings demonstrate that oxygen exposure blunts the developmental increase of CD73 postnatally in newborn mice.

To confirm the effect of oxygen exposure on CD73 transcription and protein levels, we exposed mouse lung epithelial cells (MLE-12) to oxygen and measured CD73 mRNA and protein. MLE-12 cells had decreased CD73 mRNA expression in $75 \%$ oxygen at 24,48 , and $72 \mathrm{~h}$ exposure (Figure 3c). CD73 protein levels are also decreased at all three time points (Figure $3 \mathbf{d}$ ). Dose titration of oxygen concentration demonstrated a $40 \%$ reduction in protein levels in $35 \%$ oxygen for $48 \mathrm{~h}$, a $92 \%$ reduction in protein in $55 \%$ and $75 \%$ oxygen for $48 \mathrm{~h}$ (Figure $3 \mathrm{e}$ ). Taken together, these results demonstrate that oxygen exposure decreases CD73 expression and protein levels in pulmonary cells.

\section{Loss of CD73 Worsens Abnormal Alveolarization and Pulmonary Inflammation with Oxygen Exposure}

Despite decreased CD73, hyperoxia-exposed newborn mice have an increase in pulmonary adenosine levels. To evaluate the role of elevated extracellular adenosine in hyperoxic lung injury, we used $\mathrm{CD}^{-1-}$ mice to decrease adenosine production and then measured inflammation and alveolar development. CD73 is the enzyme that performs the last step of dephosphorylation in the extracellular production of adenosine from ATP. Therefore, $C D 73^{-/-}$mice are not able to generate extracellular adenosine from ATP that is released during cellular injury. We exposed $\mathrm{CD}^{-1-}$ newborn pups to $70 \%$ oxygen or room air for 14 days after birth. BALF adenosine concentrations were decreased in $\mathrm{CD} 73^{-/-}$mice in both room air and 70\% oxygen compared with those in wildtype controls (Figure 4a). Lung sections were prepared for histology (Figure 4b). $\mathrm{CD}^{-1-}$ mice had normal lung development in room air compared with wild-type controls. However, $\mathrm{CD}^{-1-}$ mice had a marked abnormal alveolar development with increased alveolar simplification and enlargement of alveolar spaces reflected in an increase in MLI and a decrease in RAC in $\mathrm{CD}^{-/-}$mice in hyperoxia when compared with wild-type hyperoxia-exposed animals.

Alterations in growth factor signaling have been recognized to have a primary role in abnormal alveolar development in BPD (4). Two important growth factors include IGF-1, which is increased in $\operatorname{BPD}(26,27)$, and KGF, which is decreased in BPD (28). We focused on these two factors because adenosine signaling can regulate production of KGF (29), and adenosine primarily signals through alterations of intracellular cAMP, which can regulate expression of IGF-1 (refs 30,31). We measured protein levels of IGF-1 and KGF in whole-lung lysate and found a significant increase in IGF-1 protein levels in $\mathrm{CD}_{73}^{-/-}$mice when compared with the levels in wild-type 


\section{Articles | Li et al.}
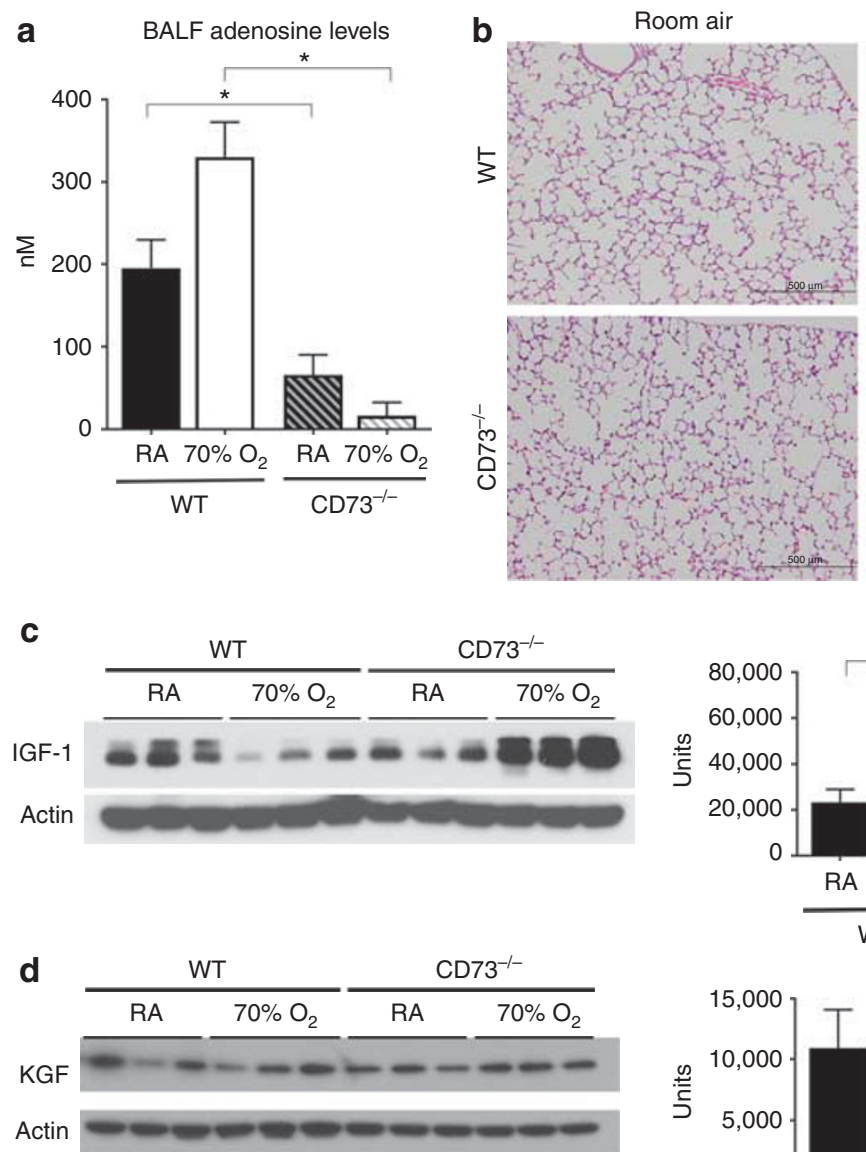
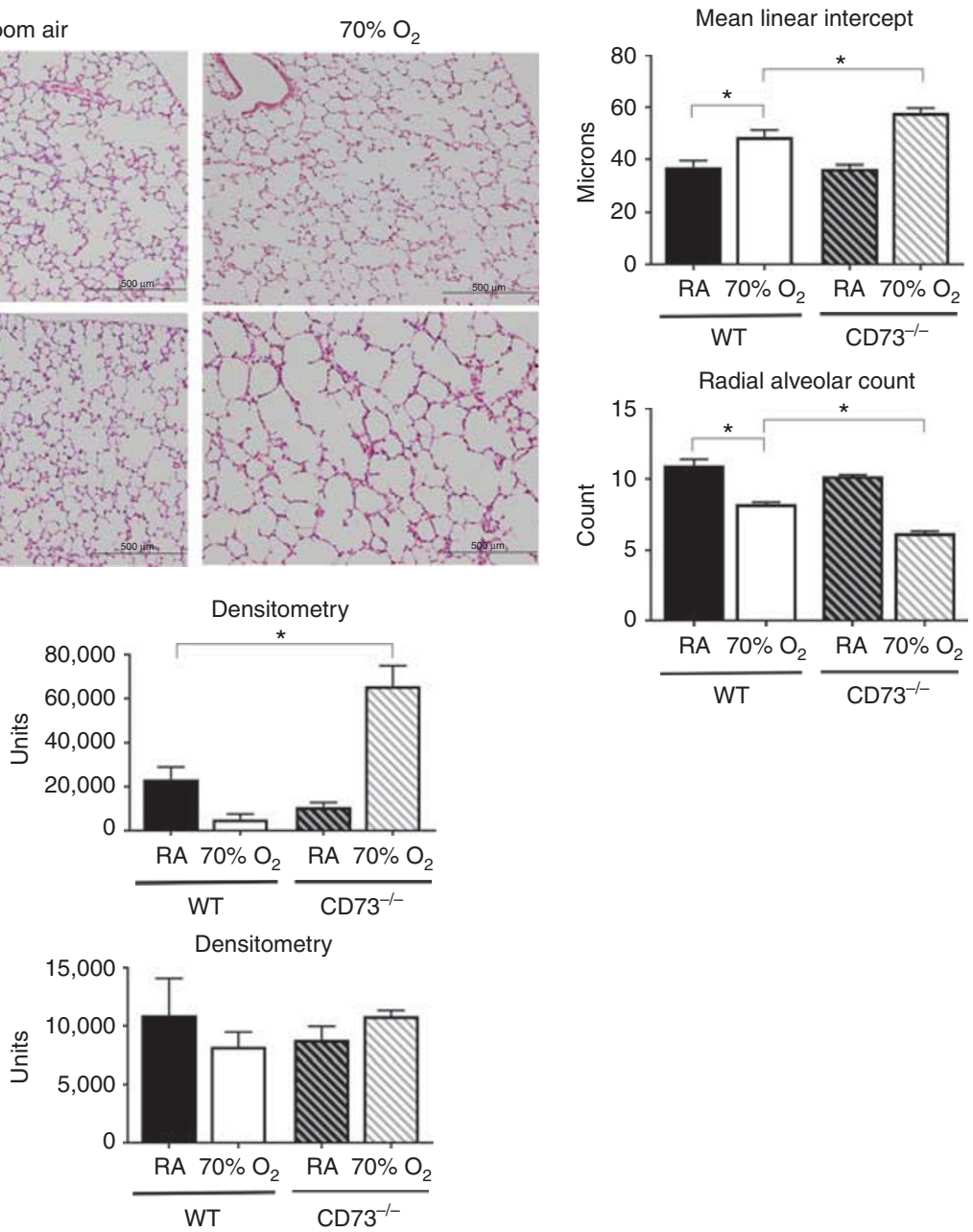

Figure 4. Loss of CD73 worsens disruption of alveolar development in $70 \%$ oxygen and increases IGF-1, but not KGF. CD73 ${ }^{-1-}$ mice were exposed starting $12-24 \mathrm{~h}$ after birth to either room air or $70 \%$ hyperoxia $\left(70 \% \mathrm{O}_{2}\right)$ for 14 days, and alveolar development was compared with wild-type mice exposed to room air and hyperoxia. (a) BALF adenosine concentrations are decreased in CD73 ${ }^{-1-}$ mice in room air and $70 \%$ hyperoxia when compared with those in wild-type mice. (b) Representative H\&E-stained histology (original magnification $\times 10$ ) and the mean linear intercept shows that loss of CD73 worsens alveolar simplification in 70\% oxygen-exposed mice. Western blot analysis shows significant increase in IGF-1 (c), but no change in $\mathrm{KGF}$ (d) in $\mathrm{CD}^{-1-}$ mice exposed to $70 \%$ oxygen. ${ }^{*} P \leq 0.05$. BALF, bronchoalveolar lavage fluid; H\&E, hematoxylin and eosin; IGIF-I, insulinlike growth factor 1; KGF, keratinocyte growth factor.

mice in hyperoxia; however, no difference in KGF protein levels was observed (Figure $4 \mathrm{~b}, \mathrm{c}$ ).

Adenosine is also a potent regulator of pulmonary inflammation following lung injury; therefore, we also evaluated inflammation with loss of CD73 in hyperoxic exposure. BALF cell counts were increased in $\mathrm{CD} 73^{-1-}$ mice in $70 \%$ oxygen exposure (Figure 5a). Differential of BALF cell types demonstrated a trend for increased macrophages, no change in neutrophils, and an increase in lymphocytes in $\mathrm{CD} 73^{-/-}$mice in hyperoxia when compared with that in wildtype controls (Figure 5b). IL-6, a known downstream target of adenosine (32) that is increased in BPD and animal BPD models $(33,34)$, was also determined. In $\mathrm{CD} 73^{-/}$mice, there was a trend for increased IL-6 in whole-lung lysate when compared with that in wild-type mice exposed to hyperoxia as measured with enzyme-linked immunosorbent assay, but the difference was not statistically significant.

\section{DISCUSSION}

Adenosine has been shown to have a regulatory role of inflammation and repair following lung injury in many adult models of lung injury (5). However, adenosine's role in the lungs of newborn animals exposed to hyperoxia remains largely unknown. Given the prevalent use of oxygen supplementation in premature neonates and its contribution to the pathophysiology of BPD, the goal of our study was to determine the role of adenosine signaling in a newborn mouse hyperoxic lung injury model of BPD. We hypothesized that hyperoxia leads to elevations of adenosine, and these elevations have a detrimental role in the developing lungs of newborn mice to disrupt alveologenesis and exacerbate pulmonary inflammation. We found that exposure to high levels of oxygen causes increased levels of extracellular adenosine despite decreasing CD73, but loss of CD73 prevented extracellular adenosine elevation, worsened 


\section{CD73 in newborn hyperoxic lung injury $\mid$ Articles}

a

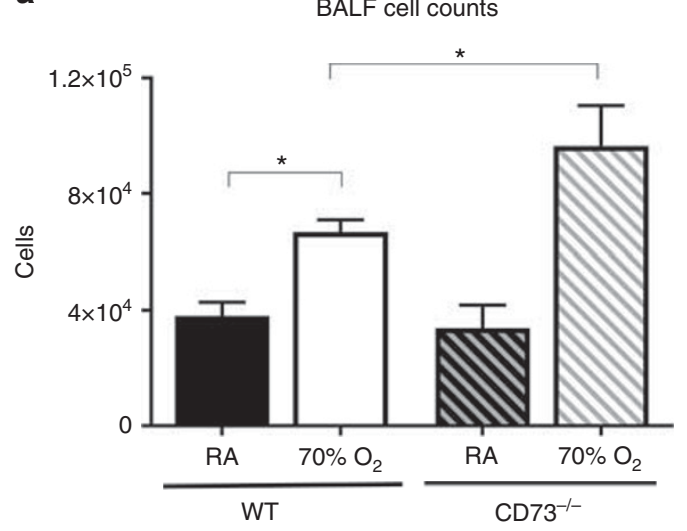

C

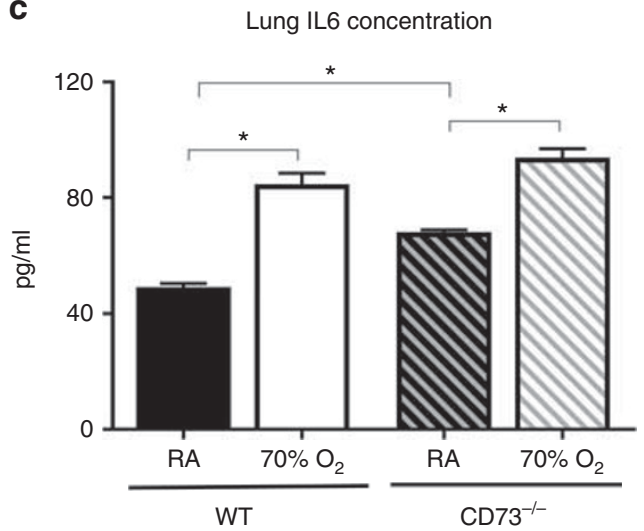

b
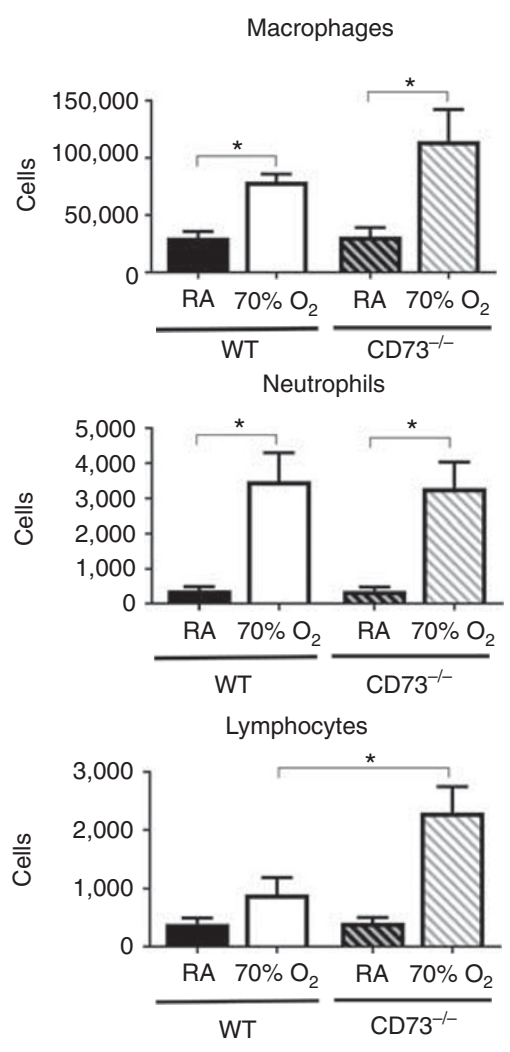

Figure 5. Loss of CD73 worsens pulmonary inflammation in $70 \%$ oxygen. (a) BALF inflammatory cell counts are increased in $\mathrm{CD} 73^{-/-}$mice when compared with those in wild-type mice in 70\% hyperoxia. (b) Cellular differentials of inflammatory cells show increases in macrophages and neutrophils in wild-type and $\mathrm{CD} 73^{-/-}$mice in $70 \%$ hyperoxia when compared with those in room air. Lymphocytes are increased in CD73 ${ }^{-/-}$mice compared with wild-type mice in 70\% hyperoxia. (c) IL-6 concentrations measured using ELISA in whole-lung lysates from wild-type and CD73 ${ }^{-/-}$ mice in room air and hyperoxia shows increased IL-6 in 70\% exposed animals. ${ }^{*} P \leq 0.05 . N=8$ for wild-type room air, 28 for wild-type hyperoxia, 10 for $\mathrm{CD}^{-/-}$room air, and 13 for $\mathrm{CD}^{-/-}$hyperoxia. BALF, bronchoalveolar lavage fluid; ELISA, enzyme-linked immunosorbent assay; IL, interleukin.

survival, and exacerbated alveolar simplification and pulmonary inflammation with hyperoxic lung injury. Taken together, our results suggest that adenosine has a protective role in hyperoxic lung injury in newborn mice.

Our first goal was to evaluate the effect of oxygen exposure on extracellular adenosine concentrations. Our in vitro and in vivo studies demonstrated a consistent downregulation of CD73 and decreased protein levels with hyperoxia exposure. This was not surprising since CD73 expression is largely controlled by the transcriptional regulation of HIF (25), and oxygen prevents it from upregulating HIF-responsive elements such as CD73 (ref. 24). However, this contradicts our findings in adult mice (9), reaffirming important differences between adults and newborns.

Despite downregulation of CD73 in hyperoxia, we found significant elevations of extracellular adenosine with exposure to both 95 and 70\% oxygen. In addition, there was a correlation between oxygen concentration, degree of inflammation, and alveolar developmental disruption and degree of adenosine elevation. This was not surprising, given the method of cellular injury in oxygen. High levels of oxygen cause oxidative damage that, along with the resulting inflammation and cell death, will lead to a large efflux of ATP from the cell that will be dephosphorylated to adenosine. The extent of cellular injury may lead to excessive ATP release that produces elevated extracellular adenosine despite downregulation of CD73. Oxidative damage to cell membranes in hyperoxia could also lead to adenosine release from the cell directly. However, the lack of adenosine elevation and phenotypic changes in $\mathrm{CD}^{-1-}$ mice in hyperoxia suggests that the CD73-mediated generation of extracellular adenosine has a primary role in regulation of tissue response following hyperoxic lung injury.

A major objective of this study was to determine the role of elevated adenosine levels in newborn hyperoxic lung injury, specifically assessing effects on alveolar development and pulmonary inflammation. A previous study from our group showed that significant pulmonary elevations of adenosine in ADA-deficient mice lead to inflammation and disrupted development (6). Lowering adenosine levels in these mice with ADA administration attenuates this pulmonary disease. On the basis of this study, we hypothesized that elevations of adenosine in hyperoxia contribute to the inflammatory 
response and abnormal alveologenesis, and decreasing extracellular adenosine production using CD73 knockout mice would attenuate the pulmonary findings in our model. Contrary to our hypothesis, the exacerbation of hyperoxic lung injury in $\mathrm{CD}^{-1} 3^{-1}$ seemingly contradicts our findings in ADA-deficient newborn mice. One possible explanation is that in ADA-deficient mice, there are also increased levels of deoxyadenosine that are known to be cytotoxic and may have detrimental effects on lung development by inducing apoptosis of key pulmonary cells (6). Alternatively, the effects could represent a goldilocks principle where ADA deficiency and $\mathrm{CD}_{73^{-/}}$produce extreme changes in extracellular adenosine levels that both produce detrimental effects. The increase in CD73 after birth suggests that adenosine signaling is tightly regulated in the developing lung, and any alteration either low or high could disrupt normal pulmonary development. We observed a correlation between oxygen level, adenosine level, and degree of disruption of alveolar development. This is supported by concentration-specific effects of caffeine on cultured AT2 cells in hyperoxia, where lower doses were protective but high doses of caffeine were deleterious (18). In addition, adenosine signaling alters newborn cytokine production in a U-shaped relationship with both low and high caffeine levels being associated with cytokines linked with BPD $(35,36)$. Finally, the Caffeine for Apnea of Prematurity trial demonstrated increased benefit from caffeine on patients receiving higher levels of respiratory support that may lead to higher adenosine levels through increased lung injury (37). Studies that further titrate adenosine levels are needed to better elucidate the relationship between adenosine signaling and disruption of alveolar development.

Despite the apparent contradictory results in our study and in ADA-deficient mice, our results are in line with a number of studies showing either no benefit or detrimental effects of caffeine, an adenosine receptor antagonist, on hyperoxiaexposed newborn mice $(15,17)$. This includes a study by Rath et al. demonstrating that caffeine had no effect on alveolar development in hyperoxia (17) and a study by Dayanim et al. showing caffeine increased inflammation and worsened disruption of alveologenesis in hyperoxia (15). Specifically, they showed that caffeine has detrimental effects on AT2 cells in hyperoxia-exposed newborn mice. Because of these findings, we evaluated KGF levels in our model, but did not find a difference in $\mathrm{CD}_{7} 3^{-/}$mice that would indicate a paracrine effect of adenosine on AT2 cells. We did find a significant increase in IGF-1 in $\mathrm{CD}^{-1-}$ mice in hyperoxia. Elevations in IGF-1 are associated with abnormal lung development in animal models of BPD (38) and human BPD lung samples $(26,39)$. Taken together, the increase in IGF-1 in hyperoxia could explain the abnormal pulmonary development in $\mathrm{CD}_{73}^{-/-}$mice. There is a well-established relationship between inflammation and alterations in lung development in the pathophysiology of BPD (40), and pathways that regulate pulmonary inflammation in the newborn lung hold important therapeutic possibilities. A key finding in our study is the exacerbation of pulmonary inflammation with increases in leukocyte recruitment by loss of CD73 in hyperoxia. This was again contradictory to previous findings in ADA-deficient mice in which sustained elevations of adenosine contributed to pulmonary leukocyte recruitment. However, it is supported by studies demonstrating adenosine's ability to alter cytokine production in neonates $(35,36)$. Perhaps, most interesting was the recognition of a U-shaped distribution in IL-1 $\beta$, IL- 6 , and tumor necrosis factor- $\alpha$ where both low and high caffeine levels were associated with higher cytokine serum concentrations (35). Thus, loss of CD73 could lead to decreased adenosine signaling similar to caffeine's antagonism of adenosine receptors, leading to increased inflammation that contributes to the abnormal alveolar development. This again suggests that the adenosine signaling pathway is tightly regulated in the newborn, and alterations too far in either direction may lead to detrimental effects.

Our findings suggesting a protective role of adenosine in hyperoxic lung injury are at odds with clinical studies showing benefits of caffeine administration. This may be because of the limitations of the animal models of human disease used in this study. Although animal models give us insight into a potentially important role for regulatory molecules such as adenosine, it is difficult to directly translate the results from our study into the clinical care of neonates, and findings in mouse models need to be validated with further studies including higher-level animals and investigations with human samples. However, we feel that our findings demonstrate the importance of understanding the role of endogenous adenosine production and metabolism on pulmonary development, and the effect of alterations in disease processes such as BPD. Specifically, this study adds to the clinical concerns raised by animal models on the use of caffeine in neonatal patients. Caffeine has been shown to decrease the risk of BPD in premature infants (14) and so many neonatal intensive care units have protocols to universally treat premature infants with caffeine regardless of respiratory support. Although the clinical data support a benefit, there may be unintentional detrimental effects that could be minimized with a better understanding of adenosine's role in the developing lung of premature neonates.

In summary, we have demonstrated that exposure to a hyperoxic environment leads to an increase in extracellular adenosine in newborn mice and loss of CD73 leads to exacerbation of abnormal alveolar development and pulmonary inflammation. These results have implications for potential therapies for prevention and treatment of BPD, as well as potential implications for the use of caffeine in premature neonates.

\section{ACKNOWLEDGMENTS}

We acknowledge Stephen E. Welty and Moorthy Bhagavatula for their assistance on the hyperoxia model.

\section{STATEMENT ON FINANCIAL SUPPORT}

This study was supported by Evangelina "Evie" Whitlock Fellowship Research Award in Neonatology (to J.D.), American Academy of Pediatrics Marshall 


\section{CD73 in newborn hyperoxic lung injury $\quad$ Articles}

Klaus Award (to J.D.), Donation by the Janet and Garth C. Bates Jr. Family (to J.D.), and National Institutes of Health grant P01-HL114457 (to M.R.B.).

Disclosure: The authors declare no conflict of interest.

\section{REFERENCES}

1. Jobe AJ. The new BPD: an arrest of lung development. Pediatr Res 1999;46:641-3.

2. Walsh MC, Szefler S, Davis J, et al. Summary proceedings from the bronchopulmonary dysplasia group. Pediatrics 2006;117:S52-6.

3. Chess PR, D'Angio CT, Pryhuber GS, Maniscalco WM. Pathogenesis of bronchopulmonary dysplasia. Semin Perinatol 2006;30:171-8.

4. Bourbon J, Boucherat O, Chailley-Heu B, Delacourt C. Control mechanisms of lung alveolar development and their disorders in bronchopulmonary dysplasia. Pediatr Res 2005;57:38R-46R.

5. Karmouty-Quintana H, Xia Y, Blackburn MR. Adenosine signaling during acute and chronic disease states. J Mol Med 2013;91:173-81.

6. Banerjee SK, Young HW, Barczak A, Erle DJ, Blackburn MR. Abnormal alveolar development associated with elevated adenine nucleosides. Am J Respir Cell Mol Biol 2004;30:38-50.

7. Luo F, Le NB, Mills T, et al. Extracellular adenosine levels are associated with the progression and exacerbation of pulmonary fibrosis. FASEB J 2015;30:874-3.

8. Zhou Y, Schneider DJ, Blackburn MR. Adenosine signaling and the regulation of chronic lung disease. Pharmacol Ther 2009;123:105-6.

9. Davies J, Karmouty-Quintana H, Le TT, et al. Adenosine promotes vascular barrier function in hyperoxic lung injury. Physiol Rep 22014.

10. Eckle T, Koeppen M, Eltzschig HK. Role of extracellular adenosine in acute lung injury. Physiology 2009;24:298-306.

11. Chunn JL, Molina JG, Mi T, Xia Y, Kellems RE, Blackburn MR. Adenosine-dependent pulmonary fibrosis in adenosine deaminasedeficient mice. J Immunol 2005;175:1937-46.

12. Kumral A, Tuzun F, Yesilirmak DC, Duman N, Ozkan H. Genetic basis of apnoea of prematurity and caffeine treatment response: role of adenosine receptor polymorphisms. Acta Paediatr 2012;101:e299-303.

13. Canpolat FE, Yurdakök M, Korkmaz A, Yiğit S, Tekinalp G. Adenosine deaminase levels in premature infants with respiratory distress syndrome and bronchopulmonary dysplasia. J Matern Fetal Neonatal Med 2011;24:703-7.

14. Schmidt B, Roberts RS, Davis P, et al. Caffeine therapy for apnea of prematurity. N Engl J Med 2006;354:2112-21.

15. Dayanim S, Lopez B, Maisonet TM, Grewal S, Londhe VA. Caffeine induces alveolar apoptosis in the hyperoxia-exposed developing mouse lung. Pediatr Res 2014;75:395-402.

16. Li J, Li G, Hu JL, et al. Chronic or high dose acute caffeine treatment protects mice against oleic acid-induced acute lung injury via an adenosine A2A receptor-independent mechanism. Eur J Pharmacol 2011;654:295-303.

17. Rath P, Nardiello C, Solaligue DE, et al. Caffeine administration modulates TGF-beta signaling but does not attenuate blunted alveolarization in a hyperoxia-based mouse model of bronchopulmonary dysplasia. Pediatr Res 2017;81:795-805.

18. Tiwari KK, Chu C, Couroucli X, Moorthy B, Lingappan K. Differential concentration-specific effects of caffeine on cell viability, oxidative stress, and cell cycle in pulmonary oxygen toxicity in vitro. Biochem Biophys Res Commun 2014;450:1345-50.

19. Volmer JB, Thompson LF, Blackburn MR. Ecto-5'-nucleotidase (CD73)mediated adenosine production is tissue protective in a model of bleomycin-induced lung injury. J Immunol 2006;176:4449-58.

20. McGowan S, Jackson SK, Jenkins-Moore M, Dai HH, Chambon P, Snyder JM. Mice bearing deletions of retinoic acid receptors demonstrate reduced lung elastin and alveolar numbers. Am J Respir Cell Mol Biol 2000;23:162-7.
21. Cooney TP, Thurlbeck WM. The radial alveolar count method of Emery and Mithal: a reappraisal 1-postnatal lung growth. Thorax 1982;37:572-9.

22. Rogers LK, Tipple TE, Nelin LD, Welty SE. Differential responses in the lungs of newborn mouse pups exposed to $85 \%$ or $>95 \%$ oxygen. Pediatr Res 2009;65:33-8.

23. Berger J, Bhandari V. Animal models of bronchopulmonary dysplasia. The term mouse models. Am J Physiol Lung Cell Mol Physiol 2014;307: L936-47.

24. Hatfield SM, Kjaergaard J, Lukashev D, et al. Systemic oxygenation weakens the hypoxia and hypoxia inducible factor 1alpha-dependent and extracellular adenosine-mediated tumor protection. J Mol Med 2014;92: 1283-92.

25. Synnestvedt K, Furuta GT, Comerford KM, et al. Ecto-5'-nucleotidase (CD73) regulation by hypoxia-inducible factor-1 mediates permeability changes in intestinal epithelia. J Clin Invest 2002;110:993-1002.

26. Chetty A, Andersson S, Lassus P, Nielsen HC. Insulin-like growth factor1 (IGF-1) and IGF-1 receptor (IGF-1 R) expression in human lung in RDS and BPD. Pediatr Pulmonol 2004;37:128-36.

27. Chetty A, Nielsen HC. Regulation of cell proliferation by insulin-like growth factor 1 in hyperoxia-exposed neonatal rat lung. Mol Genet Metab 2002;75:265-75.

28. Danan C, Franco ML, Jarreau PH, et al. High concentrations of keratinocyte growth factor in airways of premature infants predicted absence of bronchopulmonary dysplasia. Am J Respir Crit Care Med 2002;165:1384-7.

29. Ino M, Ehama R, Nakazawa $Y$, et al. Adenosine stimulates fibroblast growth factor-7 gene expression via adenosine A2b receptor signaling in dermal papilla cells. J Invest Dermatol 2007;127:1318-25.

30. McCarthy TL, Thomas MJ, Centrella M, Rotwein P. Regulation of insulin-like growth factor I transcription by cyclic adenosine $3^{\prime}, 5^{\prime}$ monophosphate (cAMP) in fetal rat bone cells through an element within exon 1: protein kinase A-dependent control without a consensus AMP response element. Endocrinology 1995;136:3901-8.

31. Thomas MJ, Umayahara $\mathrm{Y}$, Shu $\mathrm{H}$, Centrella $\mathrm{M}$, Rotwein $\mathrm{P}$, McCarthy TL. Identification of the cAMP response element that controls transcriptional activation of the insulin-like growth factor-I gene by prostaglandin E2 in osteoblasts. J Biol Chem 1996;271:21835-41.

32. Pedroza M, Schneider DJ, Karmouty-Quintana H, et al. Interleukin-6 contributes to inflammation and remodeling in a model of adenosine mediated lung injury. PLoS ONE 2011;6:e22667.

33. Ambalavanan N, Carlo WA, D'Angio CT, et al. Cytokines associated with bronchopulmonary dysplasia or death in extremely low birth weight infants. Pediatrics 2009;123:1132-41.

34. Kazzi SN, Romero R, McLaughlin K, Ager J, Janisse J. Serial changes in levels of IL-6 and IL-1beta in premature infants at risk for bronchopulmonary dysplasia. Pediatr Pulmonol 2001;31:220-6.

35. Chavez Valdez R, Ahlawat R, Wills-Karp M, Nathan A, Ezell T, Gauda EB. Correlation between serum caffeine levels and changes in cytokine profile in a cohort of preterm infants. J Pediatr 2011;158:57-64 64 e51.

36. Chavez-Valdez R, Wills-Karp M, Ahlawat R, Cristofalo EA, Nathan A, Gauda EB. Caffeine modulates TNF-alpha production by cord blood monocytes: the role of adenosine receptors. Pediatr Res 2009;65:203-8.

37. Davis PG, Schmidt B, Roberts RS, et al. Caffeine for apnea of prematurity trial: benefits may vary in subgroups. J Pediatr 2010;156:382-7.

38. Albertine KH. Progress in understanding the pathogenesis of BPD using the baboon and sheep models. Semin Perinatol 2013;37:60-8.

39. Olave N, Lal CV, Halloran B, et al. Regulation of alveolar septation by microRNA-489. Am J Physiol Lung Cell Mol Physiol 2016;310: L476-87.

40. Kallapur SG, Jobe AH. Contribution of inflammation to lung injury and development. Arch Dis Child Fetal Neonatal Ed 2006;91:F132-5. 